\title{
Identification of the Most Specific Information that a Signal Carries by Using Barwise-Seligman Theory of Information Channel
}

\author{
Rashmi Mantri \\ Database Research Group, School of Computing \\ High street, PA1 2BE \\ University of the West of Scotland, UK \\ E-mail: Rashmi.mantri@uws.ac.uk \\ Junkang Feng (Corresponding author) \\ Database Research Group, School of Computing \\ High street, PA1 2BE \\ University of the West of Scotland, UK \\ $\&$ \\ Business College, Beijing Union University, China \\ E-mail: Junkang.feng@uws.ac.uk
}

This work is partly sponsored by the a grant for Distributed Information Systems Research from the Carnegie Trust for Universities of Scotland in 2007, a grant for research on Semantic Interoperability between Distributed Digital Museums from the Carnegie Trust for Universities of Scotland in 2009 to the corresponding author, and a PhD studentship from the University of the West of Scotland, UK to the first author.

\begin{abstract}
It is a renowned phenomenon that a sign or a signal is a representation of information. Moreover Drestke claims that a sign or signal can carry only one piece of information in 'digital form' and any other in 'analogue form' in that the latter is nested in the former. After having checked in literature, a problem is dawn to us, namely no one seems to have explained how such pieces of information may be identified. We believe that it is desirable to address this problem at least as far as information systems are concerned. This is because, once identified, only such information needs to be represented explicitly and all others would be automatically represented as a result. This may applicable to database design, knowledge discovery and semantic level interoperability in the IT field, and also other fields, such as production, manufacturing and quality control where the Pareto principle could be applied. Our approach is based upon a mathematical theory of information flow, namely Barwise and Seligman's information channel theory through a case study on commonly seen traffic light. A channel is formed and the question in problem is addressed by defining the notions of classification, infomorphism, state spaces, core of the channel, constraints and serial composition of the channel.

Keywords: Information flow, Database design, Information nesting, Sign, Analogue signal, Digital signal, Information system development, Information channel theory

\section{Introduction}

A sign/signal (used as synonyms in this paper following (Mingers, 1995)) by definition carries information for its users (Feng \& Wang, 2009)(Stamper, Liu, Hafkamp, \& Ades, 2000). It has been said in literature that semiotics is useful for information system development as it helps explore the nature of signs and relations between them (Stamper et al., 2000)(Liu, 2000), (Sebeok, 2001). A signal inevitably carries more than one piece of information, and the information that is carried by a signal may be involved in such a relationship that one's existence entails that of another. This is termed 'information nesting' in the literature (Dretske, 1999). It is claimed that a signal can carry only one piece of information in 'digital form' and any other piece is in 'analogue form' in that the latter is nested in the former, and the former is termed 'the most specific information' that a signal carries(Dretske, 1999), (Mingers, 1995). We observe that it is desirable to identify such information as we would then only need to explicitly represent it and the rest would be automatically represented.
\end{abstract}


This should have positive implications to the design of an information systems where reduction in data redundancy thus optimization is desirable (Connolly \& Begg, 2005)(Batini, Ceri, \& Navathe, 1991)(Rob \& Coronel, 2009). Furthermore the long standing challenge of semantic level heterogeneity is possibly due to the fact that the knowledge discovered by different people from the same database is actually different (Halevy, 2005)(Wang, 2008)(Prasenjit Mitra, Advisor Professor, \& Gio Wiederhold, 2009)(Ouksel \& Sheth, 1999). Keeping this scenario in mind if somehow we can find the most specific information during the knowledge discovery process then we could contribute towards achieving the semantic level interoperability of databases, as semantic alignment at one level may imply alignment at others due to nesting. Supporting our claim with Pareto principle (i.e., the 80-20 rule, law of vital few) (Koch, 2005) we find that not only software applications but also production, manufacturing and quality control are identified as possible application areas of this finding.

The Pareto principle, the law of vital few also called 80-20 rule states that approximately $80 \%$ of effects come from 20\% of the causes. J.M. Muran (Bunkley, 2008) gives its name after an Italian economist V. Pareto (Amoroso, 1938) who found that $80 \%$ of the Italy's land is owned by $20 \%$ of the population. Following Pareto principle in our case we try to establish that $80 \%$ of information is represented by that of $20 \%$ which is the most specific and in digital form, that is, the $80 \%$ could be inferred from the $20 \%$. That is, we may apply our findings to wherever Pareto principle could be applied, e.g., production, economics and manufacturing etc.

However, no one including (Dretske, 1999), (Stamper, 1997),(Stamper et al., 2000) and (Liu, 2000) seems to have said about how the most specific information that a sign/signal carries may be systematically identified with mathematical rigor. We explore how this may be done as we think it is desirable to include this phenomenon in the properties of sign (Stamper, 1997),(Stamper et al., 2000). Our idea is to identify information flow in a distributed system (Barwise \& Seligman, 1997). Although Kalfoglou and Schorlemmer's contribution (Kalfoglou \& Schorlemmer, 2003), (Schorlemmer \& Kalfoglou, 2005)(Kalfoglou \& Schorlemmer, 2010) in information flow based theories is significant, they do not seem to have approached such a problem either.

What flows is captured by constraints of the form of $\Gamma \vdash \Delta$ where both $\Gamma$ and $\Delta$ are a set of types. All these terms will be presented shortly. For now we would just like to say that in $\Gamma \vdash \Delta$ the former formulates a signal, which carries the information of the existence of the latter. All the $\Delta$ 's in constraints of the form of $\Gamma \vdash \Delta$ except those that are carried by themselves in addition to $\Gamma$ would be, combined, the most specific information that $\Gamma$ carries

The above idea is based on our observation that it is possible for a signal to carry information only because there is a certain link between the signal and some information source. For example, a red traffic light links to 'the instruction to the traffic', which happens to be 'to stop' at some point of time. Such a link is termed 'information flow' in semantic theories of information (Dretske, 1999), (Barwise \& Seligman, 1997), (Keith, 1995). Information flow occurs between different components of a distributed system, and a signal carries more than one piece of information only because it is involved in more than one information flow.

Barwise-Seligman's theory enables us to approach our research question with the rigor desired. The theory uses the notion of distributed systems to formulate the situation where groups of things link with one another to form a whole, and uses the notion of information flow to formulate the relationships between these groups in terms of the existence of some element of one group entailing that of an element in another group. For example, the working of a traffic light is a group, and instructions to traffic is another group, and the former being 'red' makes certain that the latter being 'to stop'. The groups are formalised with the notion of classification, and the classifications are connected with one another through infomorphisms (which are a pair of contra-variant functions with certain property - see shortly) between classifications, whereby an information flow channel (simply channel sometimes) is formed. The channel captures why information flows between groups, and constraints formulate what flows within a channel (Barwise \& Seligman, 1997).

We construct a channel for our running example gradually in further sections followed by the notion of serial compositions of information channels. Section 2 is concerned with 'Classification'. In Section 3 'Infomorphism' is discussed. Section 4 is on 'State Spaces'. The notion of the 'Core' of a channel is described in Section 5. 'Constraints' are looked at, in Section 6. 'Serial composition of channels and Information Nesting' is described in Section 7 and Finally Section 8 includes concluding remarks.

\section{Classifications}

We build a channel (which is a mathematical model) for our system. As said earlier, a channel is made of classifications. Our system is made up of the 'Instructions to the Traffic' as a component, 'The Traffic Light' and 'The Road Situation' as other components based upon how the traffic light system works physically. These components are formulated mathematically as classifications. 
Definition 1 (Barwise \& Seligman, 1997, p.28)

A classification $\mathrm{A}=<\mathrm{A}, \Sigma_{\mathrm{A}}, F_{\mathrm{A}}>$ consists of a set A of objects to be classified called tokens, a set $\Sigma_{\mathrm{A}}$ to classify the tokens called the types of $\mathrm{A}$, and a binary relation $F_{\mathrm{A}}$ between $\mathrm{A}$ and $\Sigma_{\mathrm{A}}$, which tells one which tokens are classified as being of which type.A classification is depicted by means of a diagram in fig 1 .

A token is of a type. In the most general sense though, a 'token' is something that is being classified, and a 'type' is something that is used to classify something else. Now we could construct classifications based on the regularities of our traffic light system:

\subsection{The Instructions to the Traffic Classification $A_{\text {ItoT }}$}

The Instructions to the Traffic Classification could be classified in many different ways, the tokens are actual instances of The Instructions to the Traffic Classification ItoT, ItoT'.... at different times, and the states in which the instructions could be, say TO STOP, TO GO, RELEASE BREAK SLOWLY, MOVE SLOWLY, TO START MOVE AHEAD etc. are formulated as types.

\subsection{The Traffic Light Classification $A_{T L}$}

Similarly, we would have instances of the traffic light. The tokens are actual instances of the traffic light, i.e., TL, TL'.... at different times and the types may be RED, GREEN, AMBER, and RED AND AMBER etc.

\subsection{The Road Situation Classification $A_{R S}$}

For this classification, the tokens are actual situations on the road, i.e. RS, RS' '... at different times, and the types may be represented with the reference of the stop line as follows:

- The type of 'VEHICLE' refers to the situation where there are some vehicles present before the stop line. Similarly,

- $\quad$ 'NO VEHICLE' = no any vehicle before stop line, which means that it is clear;

- $\quad$ 'PEDESTRIAN' = pedestrian is crossing the road.

- 'NO PEDESTRIAN' = no pedestrians are around.

The concept of type and token leads to the second principle of information flow:

Second Principle of Information Flow: Information flow crucially involves both types and their particulars. (Barwise \& Seligman 1997, p.27)

Tokens or particulars (in Barwise \& Seligman 1997 terms) are important notions to consider the tokens (some individuals) in the real world are the information carriers. And the information that is carried by a token is in the form of a token being of a type. So for our traffic light example, types are $\Sigma_{\mathrm{A}}=$ \{RED, GREEN, AMBER, RED AND AMBER $\}$, and A are all the tokens of that traffic light, for example 'the traffic light at 1300 ', 'the traffic light at 1501 ', 'the traffic light at 1202 ', the relation $F_{\mathrm{A}}$ allows to determine the type of each token for the classification, for example,

'The traffic light at 1300 ' $F_{\mathrm{A}}$ RED if the traffic light is RED at 1300.

Most importantly, a token belonging to a type is the information being carried (Barwise \& Seligman 1997, p.29). This is vital and the whole theory underlies this fact.

\subsection{Classification A (The Core Classification)}

Classification A, that combines our all three classifications, i.e. The Traffic Light, the Instructions to the Traffic and The Road Situation, is a model of the whole distributed system, i.e., The Working Traffic Light System. The tokens of this classification are tuples of the tokens of the component classifications, i.e. The Traffic Light, the Instructions to the Traffic and The Road Situation that are involved in the working of The Traffic Light System, denoted $<$ ItoT, TL, RS>. The Traffic Light that is of the type BROKEN or OUT OF ORDER, not involved in the working of The Traffic Light System. The types of the core classification are the disjoint union of the types from the component classifications that are involved in the working of The Traffic Light System.

\section{Infomorphisms}

We use something called infomorphism to link up and therefore move between the component classifications

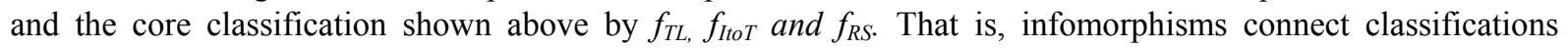
including the component classifications and the core classification to form a channel.

Definition 2 (Barwise \& Seligman, 1997, p.32) 
An infomorphism is a pair of functions that allows to map the types from component to those of the core classification, generally known as the $\mathrm{f}^{\wedge}$ (pronounced f-up), and map the tokens from the core classification to those of the component classification, generally known as the $\mathrm{f}$ function (pronounced f-down). A pair of such functions forms an infomorphism if the fundamental property of infomorphisms is satisfied. Diagrammatically, if we have two classifications $\mathbf{A}=<\mathrm{A}, \Sigma_{\mathrm{A}}, k_{\mathrm{A}}>$ and $\mathbf{C}=<\mathrm{C}, \Sigma_{\mathrm{C}}, k_{\mathrm{C}}>$, then this could be represented as shown in fig 2 .

For the pair of functions $\mathrm{f}=\left\langle\hat{\mathrm{f}}, \mathrm{f}^{\sim}>\right.$ to be an infomorphism, they must satisfy the fundamental property of infomorphisms, stated mathematically:

$\mathrm{f}^{\sim}(c) k_{\mathrm{A}} \alpha$ iff $\left.\mathrm{c}\right|_{\mathrm{C}} \mathrm{f}^{n}(\alpha)$

Where $\mathrm{c}$ is a token of the core classification and $\alpha$ is a type of the component classification (Barwise \& Seligman 1997, p.32). For the infomorphism to hold, it has to be the case where a token in the core classification must correspond (using the function $\mathrm{f}^{\sim}$ ) to at most one token of the component classification, and a type of the latter must correspond to at most one type of the core classification (using the $f^{\wedge}$ function) to which the original token of the core belongs, and vice versa.

The important point here is that infomorphisms allow us to investigate the manner in which the parts of a system fit together to facilitate the flow of information. The key notion is that of an information flow channel (explained later).

Now we look at how a channel may be constructed for our example. A natural way to proceed seems to be by defining the state space for each of the classifications, and then its infomorphism to an event classification of the state space.

\section{State Space}

Definition 3 (Barwise \& Seligman, 1997 p.46)

A state space consists of a set $\mathrm{S}$ of tokens, a set $\Omega$ of states, and a function state: $\mathrm{S} \rightarrow \Omega$ assigning a state to each token. We call $\mathrm{S}$ a complete state space if every state $\sigma \in \Omega$ is the state of some $\mathrm{S} \in \mathrm{S}$ as shown in fig 3 . A state space gives us a classification, called an event classification $\operatorname{Evt}(S)$ of $\mathrm{S}$. The tokens of $\operatorname{Evt}(S)$ are the same as $\mathrm{S}$ but it differs in types, i.e., the types of this classification are sets of states from $\mathrm{S}$, interpreted disjunctively.

\subsection{The Instructions to the Traffic state space $S_{\text {ItoT }}$}

The tokens ItoT, ItoT' .. of $\mathrm{S}_{\text {ItoT }}$ consists of the Instructions to the Traffic at different times. The real numbers between 1 and 0 are used to represent the states. These states are representing the Instructions to the Traffic. This could be any instructions between TO STOP and TO GO. We use states $_{\text {SItoT(ItoT) }}=0$ to mean to stop, 0.1 could be getting ready to go, 0.5 could be slowly move ahead, and 1 could be to go. The event classification Evt $\left(S_{\text {ItoT }}\right)$ has all the tokens of classification $\mathrm{A}_{\text {ItoT }}$ and types are subsets of the closed, unit interval $[0,1]$.

\subsubsection{Infomorphism from $\mathrm{A}_{\text {ItoT }}$ to $\operatorname{Evt}\left(\mathrm{S}_{\text {ItoT }}\right)$}

The classification $A_{\text {ItoT }}$ is actually subjective. What is considered as TO STOP, TO GO, RELEASE BREAK SLOWLY, MOVE SLOWLY, and TO START MOVE AHEAD could be different from different people's perspectives. It could be congestion on road, time of the day (busy, quite, day, night etc), how far from stop line and so forth, which could affect different persons view and actions. To deal with viewer-dependent perspectives, which might seem inimical while developing a mathematical theory of information flow, we identify infomorphism $g_{\text {ItoT }}: A_{\text {ItoT }} \rightleftarrows \operatorname{Evt}\left(S_{\text {ItoT }}\right)$ from $A_{\text {ItoT }}$ to $E v t\left(S_{\text {ItoT }}\right)$. There could be many different infomorphisms. We would believe that the following is reasonable and helpful, which is the identity on tokens and satisfies the following conditions on types:

- $g_{\text {ItoT }}($ TO STOP $)=\{0\}$

- $g_{\text {ItoT }}(\mathrm{TO} \mathrm{GO})=(0,1]$

- $g_{\text {ItoT }}($ RELEASE BREAK SLOWLY) is a left closed subset of $(0,1]$

Note: a left closed subset $X$ of a set $Y$ of reals is a set such that if $x \in X$ and $y \in Y$ is less then $x$, then $y \in X$.

- $\quad g_{\text {ItoT }}$ (TO START MOVE AHEAD) is a right closed subset of $(0,1]$

- $g_{\text {ItoT }}$ (MOVE SLOWLY) is a convex subset of $(0,1]$

Note: a convex subset $\mathrm{X}$ of a set $\mathrm{Y}$ of reals is a set such that if $\mathrm{x} 1, \mathrm{x} 2 \in \mathrm{X}$, and $\mathrm{y} \in \mathrm{Y}$ is between $\mathrm{x} 1$ and $\mathrm{x} 2$, then $\mathrm{y} \in \mathrm{X}$.

We use the diagram (fig 4) below to visualise these states: 
Every member of $g_{I t o}\left(\right.$ RELEASE BREAK SLOWLY) is less then every member of $g_{\text {ItoT }}$ ( MOVE SLOWLY), which in turn is less than every member of $g_{\text {Ito }}$ (TO START MOVE AHEAD).

More importantly we do not use the three sets, which are $g_{\text {Ito } T}$ (RELEASE BREAK SLOWLY), $g_{\text {ItoT }}$ (TO START

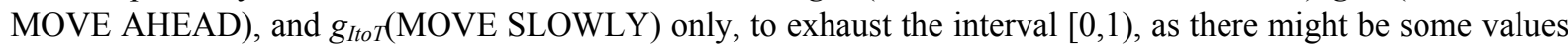
which user might be reluctant to classify appropriately.

\subsection{The Traffic Light State Space $S_{T L}$}

The tokens TL,TL'....of $S_{T L}$ consist of the traffic light at various times. Here the real numbers 0,1 and 2 are used to represent the states, showing the various states of the traffic light $\{$ RED, GREEN, AMBER $\}$. State ST $(\mathrm{tl})$ $=0$ is representing tl being RED, 1 being AMBER and 2 being GREEN. The event classification Evt $\left(S_{T L}\right)$ will have the tokens same as classification $\mathrm{A}_{\mathrm{TL}}$ and the types of this event classification will be of $\mathrm{P}(\mathrm{S})=2^{3}=8$, which is given by the power set of the state space.

$\mathrm{P}(S)=[\{$ RED, GREEN, AMBER $\},\{$ RED, GREEN $\},\{$ GREEN, AMBER $\},\{$ RED, AMBER $\},\{$ RED $\},\{$ GREEN $\}$, $\{$ AMBER $\}, \varnothing\}]=[\{0,1,2\},\{1,2\},\{3,2\},\{1,3\},\{1\},\{2\},\{3\}, \phi]$

The meaning of types of this event classification is as follows:

1) $\phi$ An empty set. An empty set is the set with no tokens of that type. It represents impossibility.

2) The type $=\{$ RED, GREEN, AMBER $\}$ or $\{0,1,2\}$ corresponds to the necessity, i.e., it is always true, as any traffic light is one of red, green or amber.

3)All other types are possible and non-necessary.

So the advantage of event classification $\operatorname{Evt}\left(S_{T L}\right)$ over $S_{T L}$ is that the former has both the impossible and necessary types as well as all other possibilities.

The token-identical infomorphism $g_{T L}: A_{T L} \rightleftarrows E v t\left(S_{T L}\right)$ could be defined so that the relationship between the types and states is clear, i.e. $g_{T L}(\mathrm{RED})=\{0\}, g_{T L}(\mathrm{AMBER})=\{1\}, g_{T L}(\mathrm{GREEN})=\{2\}, g_{T L}(\mathrm{GREEN}, \mathrm{AMBER})=\{3$, $2\}, \mathrm{g}_{T L}(\mathrm{RED}, \mathrm{AMBER})=\{1,2\}$.

\subsection{The Road situation State Space $S_{R S}$}

The tokens RS, RS'..... of $S_{R S}$ consist of the road situation at various times. Here the real numbers $0,1,2$ and 3 are used to represent the states, showing the various states of the road situation \{VEHICLE, NO VEHICLE, PEDESTRIAN, NO PEDESTRIAN $\}$. State $\mathrm{STL}_{\mathrm{ST}}(r s)=0$ represents that the road situation $r s$ is of 'VEHICLE', which means that there are some vehicles present before stop line. Similarly 1 is NO VEHICLE, 2 is PEDESTRIAN and 3 represents NO PEDESTRIAN. The event classification Evt $\left(S_{R S}\right)$ will have the same tokens as classification $A_{R S}$ and the types of this event classification will be of $P(S)=2^{4}=16$, which is given by the power set of the state space.

The token - identical infomorphism: $g_{R S}: A_{R S} \rightleftarrows E v t\left(S_{R S}\right)$ could be defined in order to find out the relationship between the types and states in a similar way of other infomorphism defined above.

\section{The information flow channel $C_{T L S}$}

The important notion of information flow channel (hereafter simplified to channel) could be explained now. Suppose there is a distributed system, modelled by means of a classification C, and several components, modelled by means of classification for $i$ in some index set $\mathrm{I}$, because $A_{i}$ is a part of $\mathrm{C}$, there must be an infomorphism $f_{i}: A i \vec{\rightleftarrows} \mathrm{C}$, one for each $i \in I$, reflecting the part-whole relationship between the system and its parts.

Definition 4 (Barwise \& Seligman 1997, p.56)

An information channel consists of an indexed family $\mathrm{C}=\left\{f_{i}: \mathrm{Ai} \rightleftarrows \mathrm{C}\right\}_{\mathrm{i} \in \mathrm{I}}$ of infomorphisms with a common codomain $\mathrm{C}$, called the core of the channel.

We have built the classification A on which the local logic $\lambda_{S}$ (see below) lives. This classification is the sum of three classifications, one for each of the components: $A=A_{T L}+A_{I t o T}+A_{R S}$. These classifications are connected by a channel as shown in fig 5.

Each of the arrows represents an infomorphism, i.e., a pair of functions, one is on the types going in and another that goes in the opposite direction, taking each token $\mathrm{c}$ to its $i^{\text {th }}$ component $a_{i}=f_{i}(\mathrm{c})$.

This is one of the most important notions of this theory. The tokens of the whole traffic light system act as a 'connection' between its various components, the various $a_{i}$. For example, a traffic light's colour is responsible for the Instructions to the Traffic in some way at a time if this kind of correspondences between them happens by 
the two of them being part of, say, a single traffic light system. Such a correspondence enables one of them to carry information about the other. This leads to the third principle of information flow.

Third principle of information flow: It is by virtue of regularities among connections that information about components of a distributed system carries information about other components (Barwise \& Seligman 1997, p.35).

Considering Figure 5 where $A_{T L}$ represents the traffic light, $A_{R S}$ the road situation, and $A_{I t o T}$ the instructions to the traffic, we can identify constraints, for example:

- GREEN, NO VEHICLE $\vdash_{\text {ctLS }}$ TO GO

- RED, NO VEHICLE $\vdash_{C T L S}$ TO STOP

Constraints formulate entailment relations between types of classifications. The above are constraints are on the core. Constraints are the major part of a local logic. The complete set of constraints of a classification is also known as a theory denoted $T h(C)$. Additionally the pair of types above $<$ (GREEN, NO VEHICLE), TO GO $>$ is also referred to as a sequent. A sequent may or may not be a constraint.

\subsection{Initial proposal}

To model the traffic light system, we start with an initial proposal. suppose that the token $a$ is of type $\alpha$, then $a$ 's being of type $\alpha$ carries the information that $b$ is of type $\beta$, relative to the channel $\mathrm{C}$, if $a$ and $b$ are connected in $\mathrm{C}$ and if the translation $\alpha^{\prime}$ of $\alpha$ entails the translation of $\beta^{\prime}$ of $\beta$ in the theory $\operatorname{Th}(\mathrm{C})$, where $\mathrm{C}$ is the core of the channel. In general this proposal works in a way that we have complete information about the regularities on the core of our channel, which is the complete theory $\operatorname{Th}(\mathrm{C})$ of $\mathrm{C}$. In the real world it seldom happens and we end up having at least some kind of commonsense theory of the core of our channel called local logic.

Definition 5 (Barwise \& Seligman 1997, p.40)

A local logic $\lambda$ consists of a classification A, a set $\vdash_{\lambda}$ of sequent's (satisfying certain structural rules) involving the types of $\mathrm{A}$, called the constraints of $\lambda$, and a subset $\mathrm{N} \subseteq \mathrm{A}$, called the normal tokens of $\lambda$, which satisfy all the constraints of $\vdash$.

Now we want a local logic on classification A and this will capture the information flow of our interest. Intuitively this local logic should have those 3 -tuples $<$ ItoT, TL, RS $>$ as tokens which include normal instances of our traffic light system, and it should have constraints as described above.

By finding the natural channel $C_{T L S}$ with our classifications $A_{T L}, A_{I t o T}$ and $A_{R S}$ as its component classifications we can now obtain a local logic associated with this system.

The information channel represents the whole traffic light system, the core of which links component classifications (i.e., The Traffic Light, The Road situation and Instructions to the Traffic) with various infomorphisms. When we model our system, we have to choose the aspects that are relevant to the problem we want to investigate. We choose to develop a state space $S_{T L S}$ and then take the core of the channel to be the event classification of this state space.

5.2 The state space $S_{T L S}$

The tokens TLS, TLS'...... of $\mathrm{S}_{\mathrm{TLS}}$ are instances of the traffic light system at various times. The set $\Omega$ of $\mathrm{S}_{\mathrm{TLS}}$ 's states is $[0,1]$, the set of the 3 -tuples of real numbers between 0 and 1 . The state can be described as $<\mathrm{r} 1, \mathrm{r} 2, \mathrm{r} 3>$ if the state of TLS's traffic light is $\mathrm{r} 1$ and the state of TLS's road situation is $\mathrm{r} 2$ and the Instructions to the Traffic is r3.

\section{Constraints on core}

In our example, from the state space of the core to that of a component classification there is a simple natural projection: from the core $S_{T L S}$ into the state spaces for the Traffic light, the Instructions to the traffic and the Road situation respectively $\left(P_{T L}: S_{T L S} \Rightarrow S_{T L,}, P_{I t T T}: S_{T L S} \Rightarrow S_{I t o T}\right.$ and $\left.P_{R S}: S_{T L S} \Rightarrow S_{R S}\right)$. These projections act as follows. Projection $P_{T L}: S_{T L S} \Rightarrow S_{T L}$, on tokens $P_{T L}$ (TLS) is the traffic light that is part of the Traffic light system instance TLS. The other projection $P_{\text {Ito } T}: S_{\text {TLS }} \rightarrow S_{\text {ItoT }}$ could be described on tokens by $P_{\text {ItoT } T}($ TLS) being instance of the Instructions to the traffic, which is the part of the traffic light system instance TLS. On states $P_{I t o T}(<\mathrm{r} 1, \mathrm{r} 2, \mathrm{r} 3>)=$ r3. With reference to states $P_{T L}(<\mathrm{r} 1, \mathrm{r} 2, \mathrm{r} 3>)=\mathrm{r} 1$. Projection $P_{R S}: S_{T L S} \Rightarrow S_{R S}$ on tokens $P_{R S}($ TLS $)$ is the road situation that is part of the Traffic light system instance TLS. With reference to states $P_{R S}(<\mathrm{r} 1, \mathrm{r} 2, \mathrm{r} 3>)=\mathrm{r} 2$. The Core of The Channel: The core of our channel $C_{T L S}$ is the event classification of $S_{T L S}$. That is, $C_{T L S}=E v t(S$ 
The infomorphism from the component classification to that of core could be defined as follows:

1) The infomorphism $f_{T L}: A_{T L} \rightleftarrows C_{T L S}$ is the composition of two infomorphisms namely,

- $g_{T L}: A_{T L} \rightleftarrows E v t\left(S_{T L}\right)$ from $A_{T L}$ into $\operatorname{Evt}\left(S_{T L}\right)$ and

- $\quad \operatorname{Evt}\left(p_{T L}\right): \operatorname{Evt}\left(S_{T L}\right) \rightleftarrows C_{T L S}$

2) The infomorphism $f_{R S}: A_{R S} \rightleftarrows C_{T L S}$ is the composition of two infomorphisms namely,

- $g_{R S}: A_{R S} \rightleftarrows E v t\left(S_{R S}\right)$ from $A_{R S}$ into $\operatorname{Evt}\left(S_{R S}\right)$, and

- $\quad \operatorname{Evt}\left(p_{R S}\right): \operatorname{Evt}\left(S_{R S}\right) \rightleftarrows C_{T L S}$

3) The infomorphism $f_{I t o T}: A_{I t o T} \rightleftarrows \mathrm{C}_{\mathrm{TLS}}$ is also the composition of two infomorphisms

- $g_{\text {ItoT } T}: \mathrm{A}_{\text {ItoT }} \rightleftarrows E v t\left(S_{I t o T}\right)$ from $A_{\text {ItoT } T}$ into $E v t\left(S_{\text {ItoT } T}\right)$, and

- $\operatorname{Evt}\left(p_{\text {ItoT }}\right): \operatorname{Evt}\left(S_{\mathrm{ItoT}}\right) \rightleftarrows C_{T L S}$

The types and the tokens of the core classification that capture the regularities of the system in which we are interested are shown in the form of a table below in fig 6 . Here the tokens are shown in the form of their states at the time $t_{1}, t_{2} \ldots . . t_{10}$ or so on and the types are the disjoint union of the types of all component classifications. From the table in Figure 6 we find the constraints on the core as follows:

- RED, VEHICLE $\vdash_{C T L S}$ TO STOP

- GREEN, VEHICLE $\vdash_{C T L S}$ MOVE SLOWLY

- RED, NO VEHICLE $\vdash_{C T L S}$ TO STOP

- GREEN, PEDESTRIAN $\vdash_{C T L S}$ TO STOP

- $\quad$ RED \& AMBER, NO VEHICLE $\vdash_{c t L S}$ TO GO

- RED \& AMBER, VEHICLE $r_{c t L S}$ TO STOP

- $\quad$ RED \& AMBER, VEHICLE $\vdash_{C T L S}$ TO STOP

- RED \& AMBER, NO PEDESTRIAN $\vdash_{\text {ctLS }}$ MOVE SLOWLEY

- RED \& AMBER, TO GO $\vdash_{\text {ct }}$ S NO PEDESTRIAN

6.1 Local logic $\lambda_{S}$

The infomorphism and classifications can be combined by what is known as '(co)limit' construction. This permits us to 'add' classifications, for example, $A_{T L}+A_{I t o T}+A_{R S}$. The tokens of this classification are the Cartesian product of all the tokens of classifications $A_{T L}, A_{I t o T}$, and $A_{R S}$, and the types are just the sum of the types of $A_{T L}$, $A_{\text {ItoT } T}$, and $A_{R S .}$. The summed classification $A_{T L}+A_{I t o T}+A_{R S}$ has the property that, given classification $\boldsymbol{C}_{T L S}$ and infomorphisms $f: A_{T L} \rightleftarrows C_{T L S}, \mathrm{~g}: \mathrm{A}_{\mathrm{RS}} \rightleftarrows \mathrm{C}_{\mathrm{TLS}}$, and $h: A_{\text {ItoT }} \rightleftarrows C_{T L S}$ there is a unique infomorphism $i=f+g+h$ such that the diagram in fig 7 commutes.

It should be noted that the relationship of $A_{T L}, A_{R S}$ and $A_{I t o T}$ to $\left(A_{T L}+A_{I t o T}+A_{R S}\right)$ are themselves, infomorphisms! That is,

$$
\begin{aligned}
& -\sigma_{\mathrm{A}}: A_{T L} \rightleftarrows A_{T L}+A_{\text {ItoT }}+A_{R S}, \\
& -\sigma_{\mathrm{B}}: A_{\text {ItoT } T} \rightleftarrows A_{T L}+A_{\text {ItoT }}+A_{R S} \\
& \text { - } \sigma_{\mathrm{C}}: A_{R S} \rightleftarrows A_{T L}+A_{\text {ItoT }}+A_{R S}
\end{aligned}
$$

We are now in a position to reason about what is going on in the core and what is going on in this new classification, i.e., $\left(A_{T L}+A_{I t o T}+A_{R S}\right)$. We have two inference rules (Barwise \& Seligman 1997, p.38) for this purpose.

$$
\begin{gathered}
f \text {-Intro : } \frac{\Gamma^{-f}-_{A} \Delta^{-f}}{\Gamma-_{B} \Delta} \\
f \text {-Elim : } \frac{\Gamma^{f}-_{B} \Delta^{f}}{\Gamma-_{A} \Delta}
\end{gathered}
$$

This is a standard notation for inference rules. That is, for example, for $f$-Intro, we have

If $\Gamma^{-f}-_{A} \Delta^{-f}$ then $\Gamma-_{B} \Delta$ must be the case.

$f$-Elim enables us to reason with constraints in the summed classification by looking at constraints of the core.

The capital gamma, $\Gamma$, and the capital delta, $\Delta$, refer to types in the classifications. These being raised to the function $f$ indicates that only those types and tokens which participate in the infomorphism should be considered. It is very important to realise this. 
Thus we can now use the local logic $\lambda_{\text {CTLS }}$ on the core $C_{T L S}$ to obtain the desired local logic $\lambda_{S}$ on the summed classification by using infomorphisms. Let $f: A \rightleftarrows C_{T L S}$ be the sum infomorphism $\Sigma \mathrm{i} \in \mathrm{I} f i$ where A is representing the summed classification $A_{T L}+A_{I t o T+} A_{R S}$ and $\mathrm{I}=\{$ ItoT, TL, RS $\}$. This infomorphism helps us 'move' the logic from $C_{T L S}$ to $A$, via $f$-Elim.

$\lambda_{S}$ is the resultant local logic. This sort of logic is denoted by $f^{-1}\left[\lambda_{C T L S}\right]$. This is the strongest logic on summed classification such that the infomorphism is well behaved. In particular $\Gamma \vdash_{\lambda s} \Delta$ will hold if and only if $f[\Gamma] \vdash$ $\lambda$ CTLS $f[\Delta]$. This local logic is complete. However, it is not sound in that it is true only for normal tokens. Normal tokens are normally only a subset of all tokens. The normal tokens consist of those tuples $<$ ItoT, TL, RS $>$ of the traffic light, road situation and the Instructions to the Traffic that are the part of the whole traffic light system $C_{T L S}$. Any $<\mathrm{ItoT}, \mathrm{TL}, \mathrm{RS}>$ that is not an instance of the whole traffic light system $C_{T L S}$ is not a normal token.

As the local logic $\lambda_{S}$ is complete, it should give us all the constraints of our interest. So, for instance if we have in

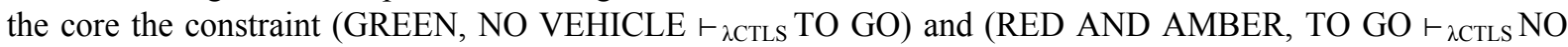
PEDETRIAN) remembering that these are types in the core and not types in the component classification, then what can we say about what is going on in summed classification $A=\left(A_{T L}+A_{I t o T}+A_{R S}\right)$ Indeed we can say:

- GREEN, NO VEHICLE $r_{\lambda S}$ TO GO, and

- RED AND AMBER, TO GO $\vdash_{\lambda s}$ NO PEDETRIAN

By definition we need to check that:

- $f_{T L}(\mathrm{GREEN}), f_{R S}$ (NO VEHICLE) $\vdash_{\lambda \mathrm{CTLS}} \quad f_{\text {ItoT }}(\mathrm{TO} \mathrm{GO})$, and

These amounts to

- $f_{T L}\left(\right.$ RED AND AMBER) , $f_{\text {Ito } T}\left(\right.$ TO GO) $\vdash_{\lambda C T L S} \quad f_{R S}$ (NO PEDETRIAN)

- $f_{T L}(\mathrm{GREEN}) \cap f_{R S}(\mathrm{NO}$ VEHICLE $) \quad \vdash f_{\text {Ito }}(\mathrm{TO} \mathrm{GO})$

- $f_{T L}($ RED AND AMBER $) \cap f_{I t o}\left(\right.$ TO GO) $\quad \vdash f_{R S}($ NO PEDETRIAN)

Using the $f$-Intro rule we can go from the component classifications to the core. Notice the types in the $f$-Intro rule are raised to the power of $-f$. The minus symbol, i.e.,-, in these situations indicates 'inverse'. This is important, because what it is saying is that only those types, in the domain of the function $f$ that result in normal tokens and types in the core classification may be considered, i.e., it is restricting to such types (and not all types of the component) to which the rule may be applied.

On the other hand, $f$-Elim allows us to move from the core classification to the summed component classifications. If a constraint is not true at the core then the sequent that corresponds to it at the component is not true either. Thus, we can say that $f$-Elim preserves non validity and therefore is complete although not sound. Both rules enable us to reason at a distance. If we have constraints in a component nearby (called the proximal), we may say anything about a remote component (called the distal).this is depicted in fig 8 .

Firstly we move from $\mathrm{P}$ to $\mathrm{C}$. As p-Intro preserves validity, the translation of a constraint of $\mathrm{P}$ on $\mathrm{C}$ would be true. Here we could say this translation is sound but not complete as there may be some constraints on $\mathrm{C}$ which we have missed by using p-intro alone. Then we move from C to D using d-Elim. As discussed above, this move would be complete but not sound as it preserves non-validity but not validity.

One could say therefore by above is that when one obtains sequent's in the distal from constraints in the proximal, the process and result is both sound and complete only as far as the tokens of the components that are involved in the tokens of the core. All bets are off for any other tokens.

\section{Serial Composition of Channels and Information Nesting}

Our basic idea for identifying the most specific information that a signal carries is to create information flow channels whereby to identity constraints and then analyse them. If we have constraints $\Gamma \vdash \Delta$ and $\Delta \vdash \Psi$, then $\Gamma$ would be the signal, and $\Delta$ would be the more specific piece of information than $\Psi$ that the signal carries. $\Gamma$ and $\Delta$ would be involved in one channel and $\Delta$ and $\Psi$ would be involved in another, and the two channels are linked through sharing one classification that includes $\Delta$. Such two channels is said a serial composition (Barwise \& Seligman, 1997), (Wang, 2008). We observe that if a signal carries two or more mutually exclusive pieces of information, then there must be at least one serial composition involved. We scrutinize channel compositions by extending the running traffic light example. Suppose we have three channels as shown in fig 9.

\subsection{Traffic Light System Channel $C_{T L S}$}

The construction of this channel is explained in above sections in detail. 


\subsection{Behaviour of the Motorist Channel $C_{B o f M}$}

The component classifications of this system are 'Instruction to the Traffic' and 'Action of the Motorist'. For the Instruction to the traffic classification, as described above it has types like TO STOP, TO GO and TO SLOW DOWN etc. For the Action of the Motorist classification, we could have types like TO BREAK FOR SLOWING DOWN, TO PUT IN THE LOWER OR UPPER GEAR, and HAND BREAK ON etc., and token of this classification will be the instances of the Instructions to the Traffic at times.

\subsection{Behaviour of the Pedestrian Channel $C_{B o f P}$}

The component classifications that make up this channel are Action of the Motorist and Action of the Pedestrian. The Action of the Motorist classification was defined above. For the classification of Action of the Pedestrian, the tokens are instances of actions of pedestrian at times and the types include TO CROSS THE ROAD, and TO STOP etc.

Behaviour of Motorist $\left(C_{B o f M}\right)$ and Behaviour of Pedestrian $\left(C_{B o f P}\right)$ are serially composed. The Instruction to the Traffic $\left(A_{I t o T}\right)$, which is our source of information and part of the component classification of traffic light system is now taking part in composition of the Behaviour of the Motorist channel and therefore this classification is a part of both the channels. There are two channels. First Channel $C_{T L S}=\left\{f_{T L}: A_{T L} \rightleftarrows C_{T L S}, f_{I t o T}: \mathrm{A}_{I t o T} \rightleftarrows C_{T L S}, f_{R S}: A_{R S} \rightleftarrows C_{T L S}\right\}$ contains connection $<\mathrm{TL}$, AofM, RS $>$ and constraint RED, VEHICLE $\vdash C_{T L S}$ TO STOP (B). Channel $\mathrm{C}_{\mathrm{BofM}}=$ $\left\{f_{\text {ItoT }(1)}: \mathrm{A}_{\text {ItoT }} \rightleftarrows \mathrm{C}_{\mathrm{BofM}}, f_{\mathrm{AofM}}: \mathrm{A}_{\mathrm{AofM}} \rightleftarrows \mathrm{C}_{\mathrm{BofM}}\right\}$ contains connection $<$ ItoT, AofM $>$ and constraints TO STOP (B) -TO BREAK FOR SLOWING DOWN (C). When we have third channel associated serially, namely Behaviour of Pedestrian with our existing channels then the component classification Instructions to the Traffic will also be taking part in the composition of this channel then this will be made up of: $\mathrm{C}_{\mathrm{BofP}}=\left\{f_{\mathrm{AofM}}(1): \mathrm{A}_{\mathrm{AofM}}\right.$ $\left.\rightleftarrows \mathrm{C}_{\text {BofP, }}, f_{\text {AofP }}: \mathrm{A}_{\text {AofP }} \rightleftarrows \mathrm{C}_{\text {BofP }}\right\}$. This contains connection $<$ AofM, AofP $>$ and constraints like TO BREAK FOR SLOWING DOWN (C) $\vdash$ TO CROSS THE ROAD (D).

From above channels we could deduce following constraints among others:

RED, VEHICLE (A) $\vdash$ TO STOP (B)

TO STOP (B) $\vdash$ TO BREAK FOR SLOWING DOWN (C)

TO BREAK FOR SLOWING DOWN (C) $\vdash$ TO CROSS THE ROAD (D)

The right hand side entails the left hand side and the former as a signal is conveying the latter as a piece of information. Moreover, information D is nested in $\mathrm{C}$ hence $\mathrm{C}$ is more specific than $\mathrm{D}$. $\mathrm{C}$ is further nested in $\mathrm{B}$ therefore less specific than B. In this example, B is the most specific information that signal A carries.

As a consequence, the construction of a serial composition of information flow channels is a sufficient condition for identifying the most specific information that a signal carries, which is achieved through the signal being modelled as a type in one classification and appearing as the left hand side of a constraint.

\section{Conclusion}

IF channel(s) enables us to reveal why information flows between a set of related things and what flows. On this basis within the scope that is captured by the channel(s) all the signals and the information that the signals carry including the most specific ones can be systematically identified. This should have positive implications to understanding databases, information systems and their design. For example, if the users know how to derive other information from the most specific ones through using nomic constraints or/and analytic constraints (Mingers, 1995), then only the latter need to be explicitly represented in an information system. That is to say, there are two points to consider when designing an information system among others. One is that different pieces of information that a system provides may be nested. The other is the ability of the user in deriving information from that which is explicitly represented. This may be measured in terms of grades of intentionality (Dretske, 1999) - the lower the less information that needs to be explicitly represented. In addition, the phenomenon of information nesting could help semantic alignment between systems in that if object $A$ is aligned with object $B$, then anything that is nested in $B$ is also aligned with $A$. Thus the discovery of the most specific information that a signal carries should help discover other information and thus help achieve more precise and accurate semantic interoperability between databases.

\section{References}

Amoroso, \& Vilfredo Pareto. (1938). Econometrica, 6(1), 21.

Barwise, J., \& Seligman, J. (1997). Information Flow: The Logic of Distributed Systems. Cambridge University Press.

Batini, C., Ceri, S., \& Navathe, S. B. (1991). Conceptual Database Design: An Entity-Relationship Approach. 
Addison Wesley.

Bunkley, \&. (2008). Joseph Juran, 103, Pioneer in Quality Control, Dies. [New York Times]. Retrieved from http://www.nytimes.com/2008/03/03/business/03juran.html

Connolly, T. M., \& Begg, C. E. (2005). Database systems: a practical approach to design, implementation, and management. Pearson Education.

Dretske, F. I. (1999). Knowledge and the Flow of Information (New edition.). Cambridge University Press.

Feng, J., \& Wang, Y. (2009). 'No representation without information flow': measuring efficacy and efficiency of representation: an information theoretic approach. W. Trans. on Comp., 8(3), 494-505.

Halevy, A. (2005). Why Your Data Won't Mix. Queue, 3(8), 50-58. doi:10.1145/1103822.1103836

Kalfoglou, Y., \& Schorlemmer, M. (2003, October). IF-Map: an ontology mapping method based on Information Flow theory. Article, . Retrieved June 3, 2010, from http://eprints.ecs.soton.ac.uk/10518/

Kalfoglou, Y., \& Schorlemmer, M. (2010). The Information-Flow Approach to Ontology-Based Semantic Integration. Book Section, . Retrieved June 3, 2010, from http://eprints.ecs.soton.ac.uk/18564/

Keith, D. (1995). Logic and Information. Cambridge University Press.

Koch, R. (2005). Living the 80/20 way. Nicholas Brealey Publishing.

Liu, K. (2000). Semiotics in information systems engineering. Cambridge University Press.

Mingers, J. C. (1995). Information and meaning: foundations for an intersubjective account. Information Systems Journal, 5(4), 285-306. doi:10.1111/j.1365-2575.1995.tb00100.x

Ouksel, A. M., \& Sheth, A. (1999). Semantic interoperability in global information systems. SIGMOD Rec., 28(1), 5-12. doi:10.1145/309844.309849

Prasenjit Mitra, Advisor Professor, \& Gio Wiederhold. (2009, July 11). Dissertation: Enabling Interoperation of Information Sources by Resolving Semantic Heterogeneity and Composing Ontologies. Retrieved from http://citeseerx.ist.psu.edu/viewdoc/summary?doi=10.1.1.141.7976

Rob, P., \& Coronel, C. (2009). Database systems: design, implementation, and management. Cengage Learning.

Schorlemmer, M., \& Kalfoglou, Y. (2005). Progressive Ontology Alignment for Meaning Coordination: an Information-Theoretic Foundation. Conference or Workshop Item, . Retrieved June 3, 2010, from http://eprints.ecs.soton.ac.uk/11556/

Sebeok, T. A. (2001). Signs: an introduction to semiotics. University of Toronto Press.

Stamper, R. (1997). Organizational semiotics: Information systems: an emerging discipline?, McGraw-Hill, London.

Stamper, R., Liu, K., Hafkamp, M., \& Ades, Y. (2000). Understanding the roles of signs and norms in organizations - a semiotic approach to information systems design. Behaviour \& Information Technology, 19(1), 15. doi: $10.1080 / 014492900118768$

Wang, Y. (2008). An Info Flow Framework of Representation and its Application for Semantic Integration (Technical report). University of the West of Scotland. Retrieved from British Library. 
$\Sigma_{A}$

$F_{A}$

Fig.1. Classification

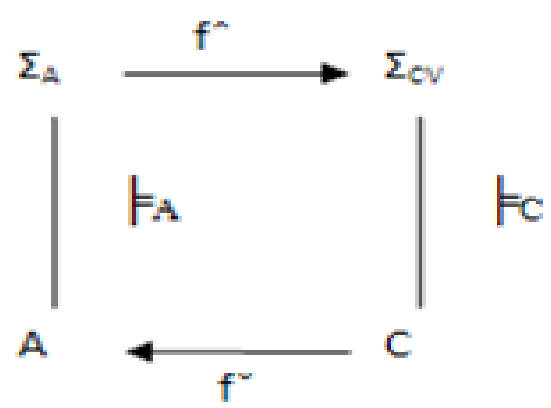

Fig.2. Infomorphisms

$\Omega$

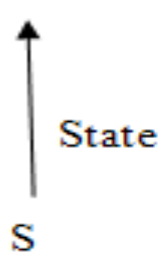

Fig.3. State Space 


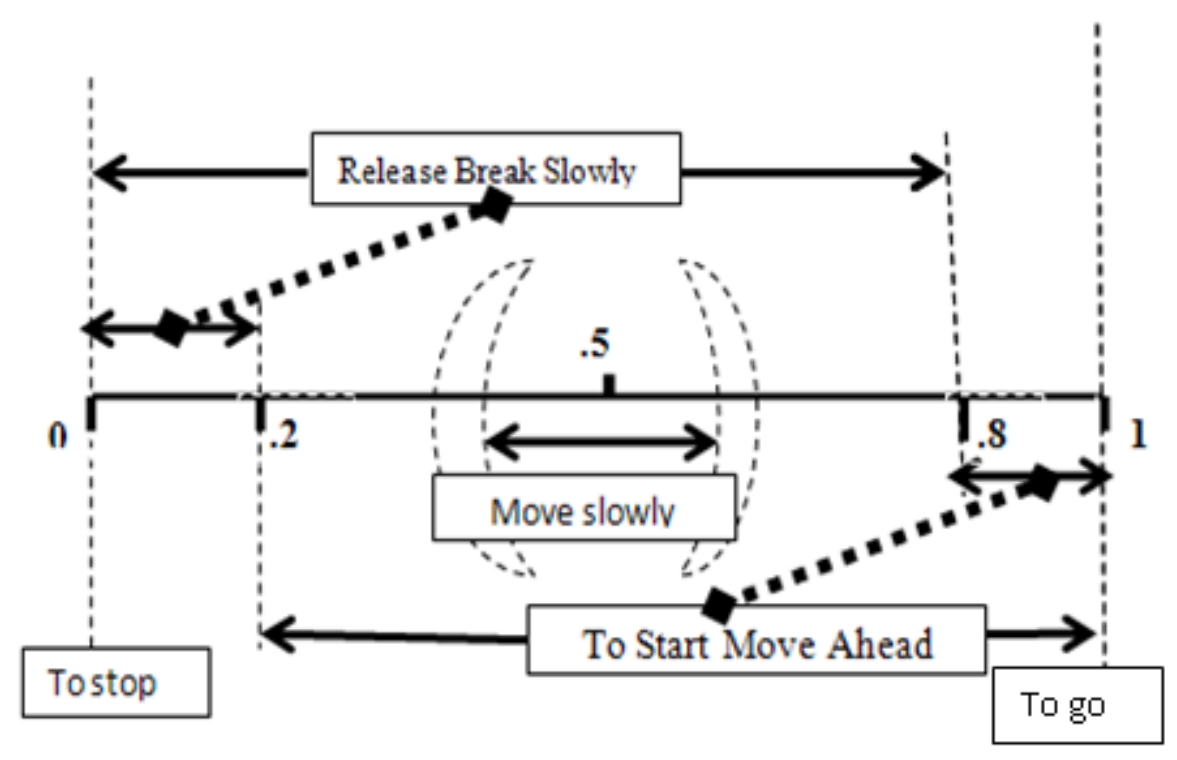

Fig.4. Ranges of types in event classification of Evt($\left(\mathrm{S}_{\mathrm{Ito}} \mathrm{T}\right)$

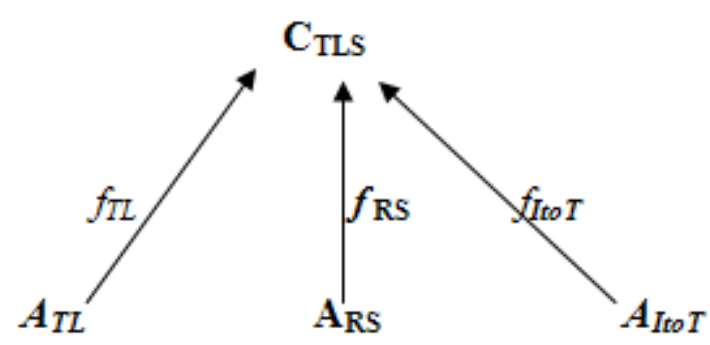

Fig.5. The Information channel $C_{\text {TLS }}$ 


\begin{tabular}{|l|l|l|l|l|l|l|l|l|l|l|}
\hline Tokens & Red & Green & Amber & Vehicl & $\begin{array}{l}\text { No } \\
\text { vehicle }\end{array}$ & pedestrian & $\begin{array}{l}\text { No } \\
\text { pedestrian }\end{array}$ & $\begin{array}{l}\text { To } \\
\text { Go }\end{array}$ & $\begin{array}{l}\text { To } \\
\text { stop }\end{array}$ & $\begin{array}{l}\text { Move } \\
\text { Slowly }\end{array}$ \\
\hline $\begin{array}{l}\text { Red, vehicle to } \\
\text { stop }\end{array}$ & $\sqrt{ }$ & & & $\sqrt{ }$ & & & & & $\sqrt{ }$ & \\
\hline $\begin{array}{l}\text { (green, vehicle, } \\
\text { move slowly) }\end{array}$ & & $\sqrt{ }$ & & $\sqrt{ }$ & & & & & & $\sqrt{ }$ \\
\hline $\begin{array}{l}\text { (red, no vehicle, to } \\
\text { stop) }\end{array}$ & $\sqrt{ }$ & & & & $\sqrt{ }$ & & & & $\sqrt{ }$ & \\
\hline $\begin{array}{l}\text { (green, pedestrian, } \\
\text { to stop) }\end{array}$ & & $\sqrt{ }$ & & & & $\sqrt{ }$ & & & $\sqrt{ }$ & \\
\hline $\begin{array}{l}\text { (red \& amber, no } \\
\text { vehicle, to go) }\end{array}$ & $\sqrt{ }$ & & $\sqrt{ }$ & & $\sqrt{ }$ & & & $\sqrt{ }$ & & \\
\hline $\begin{array}{l}\text { (red \& amber, } \\
\text { vehicle, to stop) }\end{array}$ & $\sqrt{ }$ & & $\sqrt{ }$ & $\sqrt{ }$ & & & & & $\sqrt{ }$ & \\
\hline $\begin{array}{l}\text { (red \& amber, } \\
\text { vehicle, to stop) }\end{array}$ & $\sqrt{ }$ & & $\sqrt{ }$ & $\sqrt{ }$ & & & & & $\sqrt{ }$ & \\
\hline $\begin{array}{l}\text { (red \& amber, no } \\
\text { pedestrian, move } \\
\text { slowly) }\end{array}$ & $\sqrt{ }$ & & $\sqrt{ }$ & & & & $\sqrt{ }$ & & & $\sqrt{ }$ \\
\hline $\begin{array}{l}\text { (red and amber, to } \\
\text { go, no pedestrian) }\end{array}$ & $\sqrt{ }$ & & $\sqrt{ }$ & & & & $\sqrt{ }$ & $\sqrt{ }$ & & \\
\hline
\end{tabular}

Fig.6. Classification table

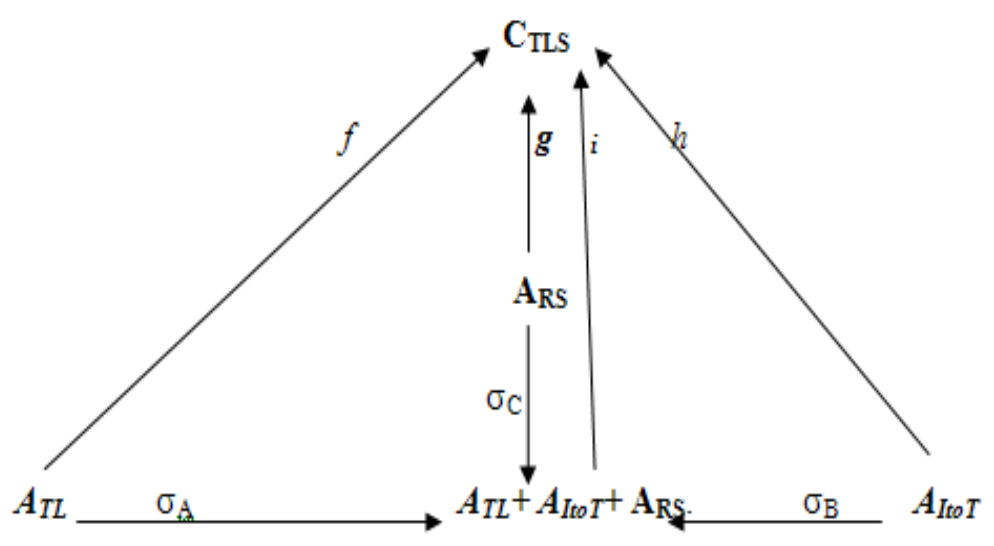

Fig.7. Summed classification 


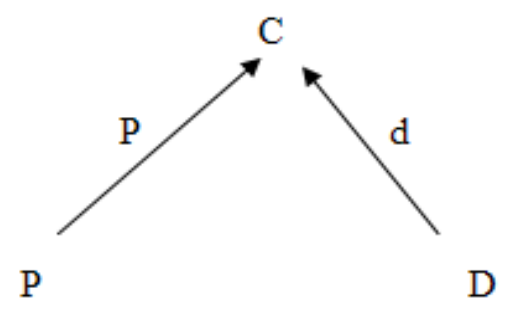

Fig.8. A channel made up of a proximal and a distal

\section{Traffic Light System}

\section{Behaviour of the motorist}

\section{Behaviour of the pedestrian}

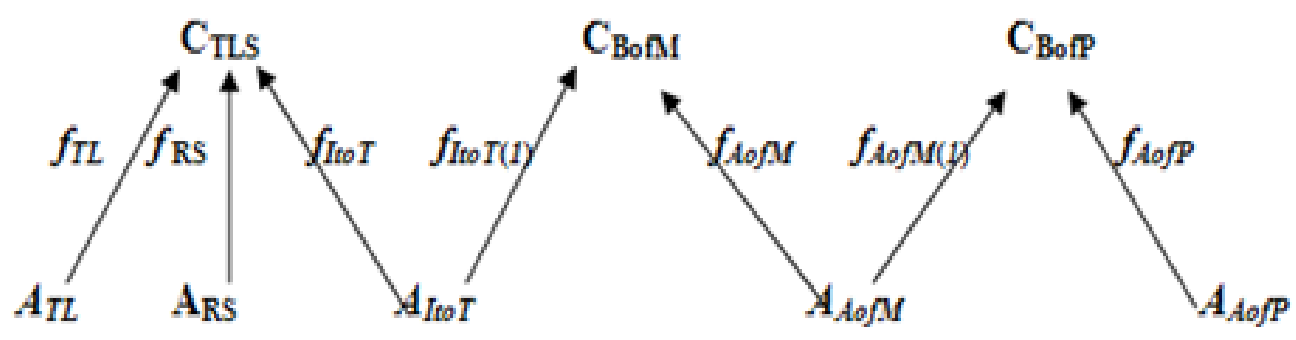

Fig.9. Serial composition of channels 